\title{
Discrimination of dynamic change and constancy over time by pigeons
}

\author{
Carl Erick Hagmann • Robert G. Cook
}

Published online: 12 April 2011

(C) Psychonomic Society, Inc. 2011

\begin{abstract}
The detection of change over time is critical to the serial integration of reality. Three pigeons, in a same/different go/no-go discrimination, were rewarded for pecking at changing stimuli that oscillated back and forth in brightness over a specific range and not at constant, unchanging stimuli randomly selected from the same range. Experiment 1 tested their capacity to detect increasingly slower rates of change against a constant control. The results indicated that pigeons retrospectively integrate past experience over approximately 20-30 s. Experiment 2 tested combinations of brightness ranges and rates to examine the possible roles of perception and memory in this discrimination. Overall, the results indicate that pigeons can detect continuous changes in brightness over different temporal durations, and several lines of evidence suggest that a combination of perception and memory mechanisms are involved. Implications for the pigeons'experience of the recent past are considered.
\end{abstract}

Keywords Pigeons · Perception · Working memory · Change detection $\cdot$ Time horizon

The stable experience of reality is critically tied to the perception and integration of change and constancy across time. Built across different temporal windows, such integration is an important part of constructing the "narrative" of experience. In humans, several mechanisms seem to be involved. The first involves direct detection by perceptual processes, such as when the perceptual system integrates movement over time (e.g., Seiffert \& Cavanagh, 1999). When this motion system breaks down, objects can disruptively

C. E. Hagmann $(\varangle) \cdot$ R. G. Cook

Department of Psychology, Tufts University,

Medford, MA 02155, USA

e-mail: Carl.Hagmann@tufts.edu appear in one location and then suddenly in another with little intervening movement (Zihl, Von Cramon, \& Mai, 1983). The integration of recent experience by memory is also critical. Change blindness experiments, in which perceptual mechanisms are eliminated by the inclusion of a brief temporal gap between alternating pictures, have focused on these short-term memory mechanisms (e.g., Rensink, 2002). When memory integration does fail, humans often resort to confabulation to help explain temporal rifts in reality (Kopelman, 1987; Sacks, 1998). Some changes occur too slowly for either perception or working memory to detect, such as the slow growth of a child or a plant. In these cases, change can be detected only by comparing long-term memories of the past with the present. It is the interaction between these perception and memory mechanisms that builds the continuous experience of reality across time (James, 1890; Metzinger, 2003; Tononi, 2004).

Nonhuman animals face analogous issues in their sequential integration of past and recent experiences. This aspect of their cognition has not been widely examined, however. Simple animals with limited working memories may rely on immediate perceptions, thus perhaps being creatures of the "moment." More complex animals likely use combinations of perception and memory to continually integrate and update their experience over longer time horizons. Different species or classes could have different time horizons for these mechanisms and, thus, could differ in their capacity to knit together their experience of "then" and "now" (e.g., Wright, Santiago, Sands, Kendrick, \& Cook, 1985). In the present study, we investigated for the first time how pigeons (Columba livia) respond to continuously changing information presented at different rates, in order to provide insight into how they organize and integrate recent experience with perceptual and memory mechanisms.

Some understanding of how animals integrate past information can be gleaned from working memory research. In 
these experiments, animals are asked to remember a discrete stimulus over variable delays. For pigeons, delays of more than $20 \mathrm{~s}$ are typically problematic (e.g., Blough, 1959; Grant, 1976; Wixted, 1989). More relevant to the goals of the present study are those from successive same/different (S/D) studies. These studies examined discrete sequences of repeatedly different (e.g., ABAB .. .) or the same (e.g., AAA ... or BBB . . .) pictorial stimuli (Cook \& Blaisdell, 2006; Cook, Kelly, \& Katz, 2003; Wasserman, Frank, \& Young, 2002; Wright et al., 1985). For instance, Cook and Blaisdell examined how long the initial item of a sequence influenced behavior by testing pigeons with repeated different sequences of varying lengths (e.g., $\mathrm{ABAB}$. . . , $\mathrm{ABCABC} \ldots . . \mathrm{ABCDABCD}$...). By varying the time between pictures, they found that memory for the first item lasted about $10 \mathrm{~s}$ and could survive several intervening different items.

To mimic the continuous nature of experience in studying change detection, we tested 3 pigeons in a novel S/D go/no-go discrimination. On same/constant trials, a randomly selected brightness value appeared unchanging within a square patch on the screen. On different/change trials, the brightness of this patch dynamically oscillated between dark and light. The successive changes in the display were made by varying its brightness in increments much smaller than the $10 \%$ luminance differences needed for pigeons to detect a difference in successive discriminations (Hodos \& Bonbright, 1972). Thus, the pigeons were required to make successive comparisons over time in order to detect change. This original aspect of having the different condition dynamically change allowed us to gain new insights into how pigeons integrate recent experiences and perceive changes in their environment.

In the first experiment, they were tested with stimuli that changed at rates easily perceived by humans (unpublished observations). Over four successive phases, the pigeons were tested with increasingly slower rates of change that would require longer working memory spans to differentiate change from constancy. With slower rates, we expected the pigeons to more frequently regard the changing stimuli as "constant." In a second experiment, we examined how perceptual and memory mechanisms might be differentially influenced by the range of tested values, as well as the rate of change. We hypothesized that separate perceptual (fast rates) and memory (slow rates) change detection mechanisms might be differentially sensitive to different ranges.

\section{Method}

Animals Three male pigeons were tested. Each had previously discriminated moving object videos. They were maintained at $80 \%-85 \%$ of their free-feeding weights, with free access to grit and water.

Apparatus Testing was conducted in a computer-controlled chamber. Stimuli were presented on an LCD monitor (ViewSonic, 1,024 $\times 768$ pixels) visible behind a $26 \times 18 \mathrm{~cm}$ infrared touchscreen (EloTouch; Harrisburg, PA) that recorded pecks. A ceiling light was illuminated at all times, except during time-outs. A central food hopper was located under the touchscreen.

Procedure Discriminative stimuli appeared as a central rectangle $(10.2 \times 16.5 \mathrm{~cm})$ on a salmon background (RGB: 255, 192, 192; used to minimize simultaneous contrast).Throughout a trial, this rectangle was either a single grayscale value (gsv) held constant or a continuously changing series of gsv in a sawtooth pattern (see Fig. 1). The 256 gsv available $(0=$ black, $255=$ white $)$ ranged in brightness from 0.31 to 37.4 candelas per $\mathrm{m}^{2}$ (Minolta Chromameter CS-100). Subsets of this total range were used in phases 1-3. Initial brightness values were randomly selected for each trial from within this subset. Because the experiment was designed around the gsv controlling the video card, the results are described in these units.

Trials began with a peck to a $2.5-\mathrm{cm}$ diameter circular green ready signal. This was replaced by a constant or changing rectangle for $24 \mathrm{~s}$. Pecks during change trials were reinforced (2.9-s grain access) on a variable-interval 10 -s schedule. Pecks during constant trials resulted in no reinforcement and a variable length time-out ( $1 \mathrm{~s}$ per peck) upon its termination. A 5-s intertrial-interval was used. To eliminate the role of reinforcement-based cues, infrequent $(<10 \%)$, nonreinforced probe trials were randomly mixed
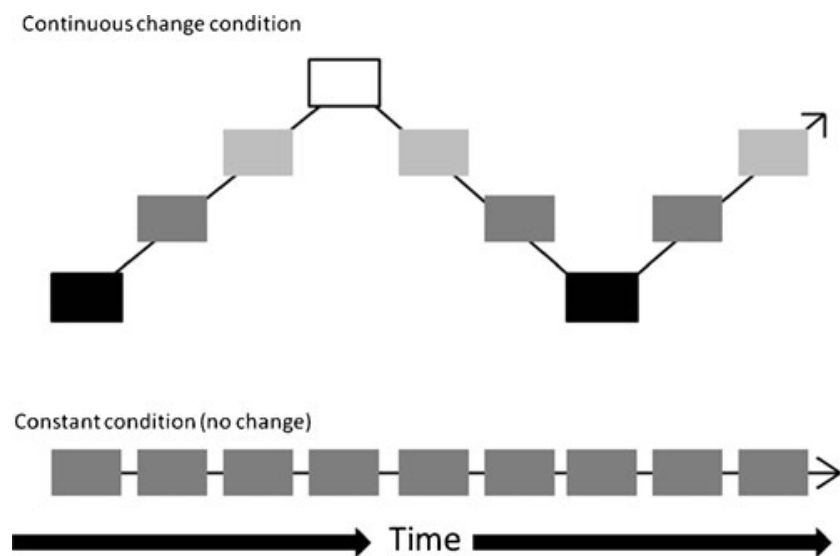

Fig. 1 Illustrative representation of the two trial types tested in these experiments. The top portion shows the gradual oscillation between brightness values during the temporal duration of a change trial $(\mathrm{S}+)$. The bottom portion shows the corresponding absence of change on constant trials $\left(\mathrm{S}^{-}\right)$ 
into each session. Probe trials were identical to change trials, except for the absence of reinforcement. Only data from constant trials and change probe trials were analyzed.

During initial discrimination training, the pigeons were tested with a range of $70 \mathrm{gsv}$. There were 72 trials per session (36 constant/36 change; 12 trials per change rate: 100, 33.3, and $20 \mathrm{gsv} / \mathrm{s}$; two probes per rate). After 50 training sessions, pigeons $1 \mathrm{~A}$ and $3 \mathrm{~S}$ discriminated all rates from constancy, while $2 \mathrm{M}$ showed little discrimination. The brightness range was expanded to $140 \mathrm{gsv}$. With this change, 2M began discriminating, and Experiment 1 began.

Experiment 1 had four phases. Phase 1 consisted of 20 sessions with the conditions used during training. Phase 2 tested two new slower rates (13.3 and $6.7 \mathrm{gsv} / \mathrm{s})$ and an established fast rate $(33.3 \mathrm{gsv} / \mathrm{s})$ for 20 sessions. There were 72 trials per session (36 constant/36 change, 12 per rate, two probes per rate). Phase 3 tested two new rates (4.4 and $3.3 \mathrm{gsv} / \mathrm{s}$ ) slower than those previously tested, one new medium rate $(10 \mathrm{gsv} / \mathrm{s})$, and the common fast rate $(33.3 \mathrm{gsv} / \mathrm{s})$ as a reference. Trial durations were extended to $40 \mathrm{~s}$. Twenty 80-trial sessions (40 constant/ 40 change; 10 per rate, one probe per rate) were conducted. Phase 4 tested two prior rates (33.3 and 4.4 $\mathrm{gsv} / \mathrm{s})$ and three new slower rates $(2.5,2$, and $1 \mathrm{gsv} / \mathrm{s})$ with the entire 256-gsv range. Only pigeons $1 \mathrm{~A}$ and $2 \mathrm{M}$ were tested in this phase. Fifty 80-trial sessions (40 constant/40 change; 8 per rate, one probe per rate) were conducted. Half the trials were extended to $60 \mathrm{~s}$. Data from all trials were used for analysis of the first $40 \mathrm{~s}$ and only from 60-s trials for times greater than $40 \mathrm{~s}$.

Experiment 2 was tested after phase 3 and included all 3 pigeons. Two rates (33.3 and $4.4 \mathrm{gsv} / \mathrm{s}$ ) and six brightness ranges $(20,40,60,80,100$, and $120 \mathrm{gsv})$ were tested for 20 sessions. Sessions consisted of the 80 trials described for phase 3 , plus 12 probe trials that tested each rate/range combination once per session.

\section{Results}

Figure 2 shows the time course of the pigeons' detection of successive brightness changes within a trial over the four phases of Experiment 1. Each panel shows mean normalized peck rate as a function of time (2-s bins) within a trial (normalized to each bird's mean maximum pecks per condition). Discrimination emerged over time as peck rates progressively declined in the constant condition relative to change conditions. In phase 1 , they easily discriminated the three rapidly changing conditions from the constant condition (upper left panel). Across the next three phases, they showed greater difficulty maintaining this level of discrimination with increasingly slower rates of change.
Their behavior on slower change trials increasingly resembled that on the constant trials (cf. three remaining panels). A repeated measures ANOVA (rate $\times$ time) of these normalized peck rates, using the common $24 \mathrm{~s}$ across all four phases, confirmed the presence of a significant rate $\times$ time interaction, $F(33,66)=4.4$ (an alpha of $\mathrm{p}<.05$ was used for all tests).

A similar repeated measures ANOVA for just phase 1 also yielded a significant rate $\times$ time interaction, $F(33,66)=4.4$. A second ANOVA comparing normalized peck rates for just the three change conditions showed no significant main effect of rate, $F(2,4)=1.03$. For phase 1 , these results indicate that rate of change made no difference and that all rates were discriminated from the constant condition. To model the shape of these functions, we fitted linear and two forms of exponential decay functions to each condition. These fits were performed on results obtainedafter the first $4 \mathrm{~s}$, because of the increase in peck rate briefly observed at the beginning of each trial. For the three change conditions, all fits converged on a linear solution, indicating no change in peck rate over the last $20 \mathrm{~s}$. For the constant condition, the best-fitting function was an exponential decay function with a parameter adjusting for asymptote $\left[F=y+a^{*} \exp \left(-b^{*} x\right)\right]$. This function provided an excellent fit, accounting for $99.7 \%$ of the variance in constant responding.

To measure when change peck rates significantly diverged from constant peck rates, we used $t$-tests comparing performance across each 2-s bin for each bird. The point of divergence was calculated to be at the first of two consecutive or two out of three significantly different bins. Requiring a series of independent significant values provided a built-in check for Type 1 errors. We further checked that these values remained different for the rest of the trial. The point of divergence for each phase 1 rate occurred at means of 4, 6, and $8.6 \mathrm{~s}$, respectively, indicating that slower change rates took just slightly longer to discriminate.

In phase 2 , there was again a significant rate $\times$ time interaction, $F(33,66)=5.4$, with the three change conditions again best fit by linear solutions and the constant condition best fit by an exponential function. Overall, peck rates for the two new slower rates were lower and diverged later from those in the constant condition ( $M \mathrm{~s}=14.6$ and $17.3 \mathrm{~s}$, respectively) than from those in the common fast condition (7.3 s) for all 3 pigeons. This difference is also reflected in the significant rate $\times$ time interaction, $F(22,44)=2.1$, present among the three change conditions.

Phase 3's results were essentially similar, although there was general decline in responding across all conditions toward the end of a trial, probably because of the longer presentations. There were significant rate $\times$ time interac- 


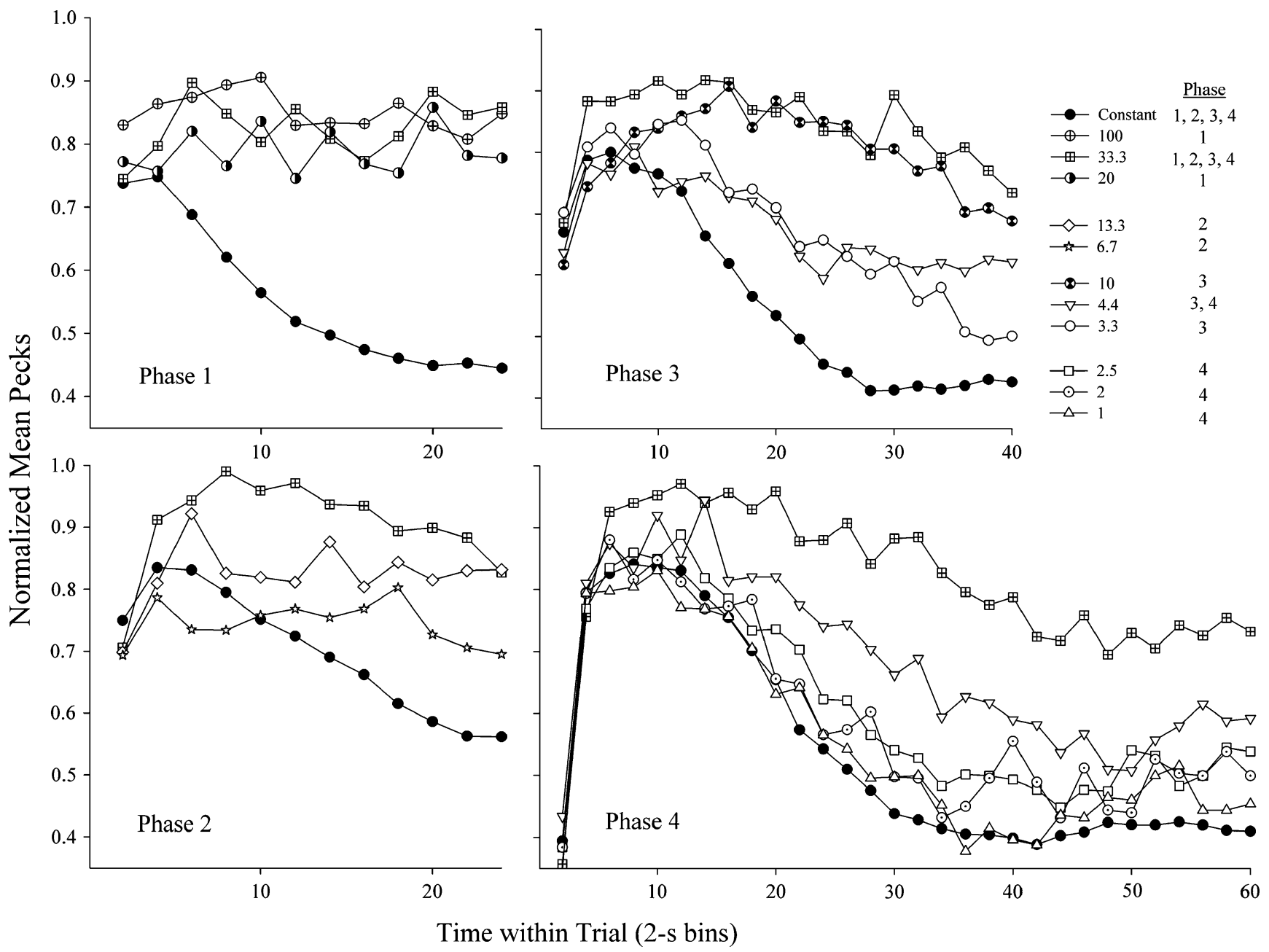

Fig. 2 Normalized mean pecks within a trial divided into 2-s bins for all sessions in the different phases of Experiment 1 . The unfilled symbols represent pecking behavior in change conditions $(\mathrm{S}+)$, and the filled circles represent pecking behavior during the constant condition (S-)

tions in analyses with, $F(76,152)=3.36$, and without, $F$ $(57,114)=2.17$, the constant condition. The two fastest rates were again best fit by a linear function, while the constant condition was best described by the exponential function. Of the two intermediate rates, one was exponential in form, while the other showed a linear decline. Mean divergence times were $8,10.6,18$, and $15.3 \mathrm{~s}$, respectively, for each rate.

In phase 4, change discrimination suffered considerably, especially for the slowest rates. The faster rates continued to show good change detection, since the main effects of both time, $F(29,29)=8.5$, and rate, $F(5,5)=20.3$, were significant. The previous rate $\times$ time interactions disappeared, since the slow change conditions now produced results resembling those in the constant condition. Mean divergence times at the first four rates were $6,14,20$, and $38 \mathrm{~s}$, respectively, for pigeon $1 \mathrm{~A}$ and $4,20,28$, and $26 \mathrm{~s}$, respectively, for $2 \mathrm{M}$. At the slowest rate, $2 \mathrm{M}$ met our divergence criterion at $30 \mathrm{~s}$, but this was not maintained, and 1A showed no divergence. Thus, neither bird could detect change at a rate of $1 \mathrm{gsv} / \mathrm{s}$. The three slowest rates were best fit exponentially, especially over the first $50 \mathrm{~s}$. The fastest rate was still best fit linearly over the first $50 \mathrm{~s}$, although the extended flat tail present at the end of each trial resulted in a better exponential fit over the entire duration

The presence of an exponential character in the functions with the slowest rates of change suggests an increasing number of such trials were treated as "constant." Thus, intermediate peck rates between the fastest change and constant extremes in the different phases may reflect mixtures of high (reporting "change") and low (reporting "constant") peck rates being averaged across trials. Evidence indicative of an "all-or-none" response pattern can be seen in Fig. 3. It shows the frequency distribution of pecking over time for each rate from phase 4 . The upper left panel shows that peck rates remained high for the entire presentation of the fastest rate. The bottom right shows the 
Fig. 3 Surface plots showing normalized frequency distributions of pecking within a trial divided into 2-s bins during phase 4 of Experiment 1. The six plots are respectively arranged for trials having fast rates of change (upper left) to increasingly slower changes to the constant condition (bottom right). Higher regions (red/light peaks) represent bins containing the greatest frequency of pecks, while the blue/dark areas show lower peck rates. With slower rates, a clear shift toward pecking less increasingly occurs in the latter half of an average trial, making the graphs increasingly similar to that for the constant condition
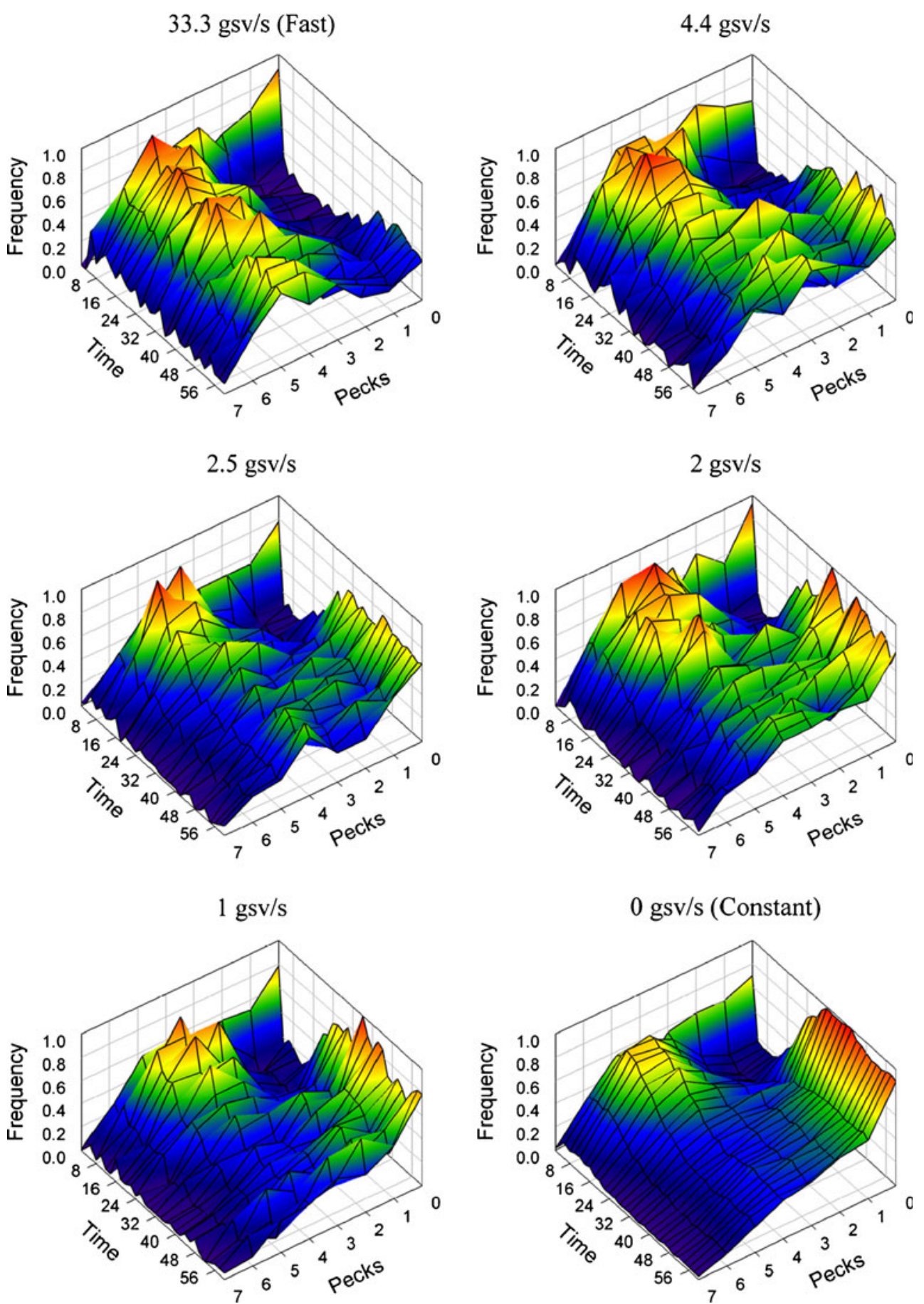

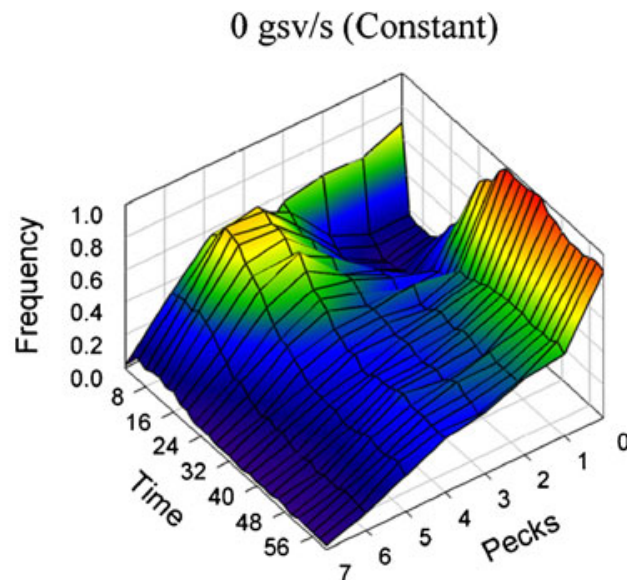

transition on constant trials from an initial burst of pecking to the cessation of pecking later in the trial. The distributions in the middle panels are diagnostically bimodal, since the pigeons pecked either rapidly or almost not at all. This pattern suggests they were, at any given point in a trial, experiencing one of two states - change or constancy-and responding appropriately. Monte Carlo modeling, not included here, confirmed that the peck distributions for the intermediate rates could be satisfacto- rily generated by probabilistic admixtures of the pecking behavior observed on fast and constant trials.

Figure 4 shows mean divergence time as a function of rate across all four phases. An ANOVA confirmed that divergence times increased as an inverse function of rate, $F(9,16)=12.8$, since they required greater time as the rate of change slowed. Perhaps not surprisingly, a logarithmic curve provided the best fit, suggesting that the psychophysics of detecting change across time shares 


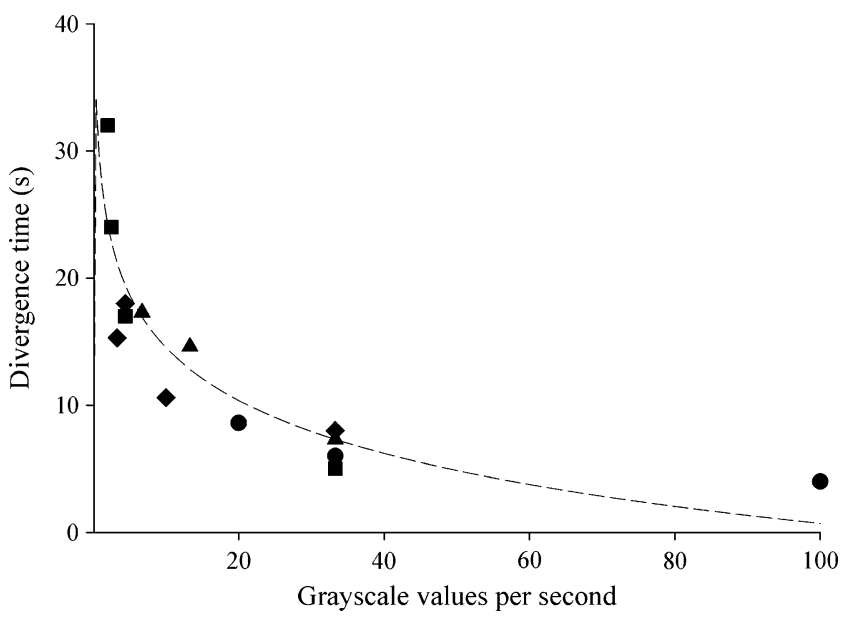

Fig. 4 Mean divergence points as a function of rate of change in Experiment 1. Circles represent divergence points obtained for phase 1 , triangles for phase 2 , diamonds for phase 3 , and squares for phase 4. The best-fitting logarithmic function is plotted and has an $R^{2}$ of .78

similarities with the findings of other studies of temporal discrimination (e.g., Roberts, 2006).

Experiment 2's results are shown in Fig. 5. We hypothesized that detection of rapid change might be heavily reliant on perception and, therefore, might require a smaller amplitude for detection than would slower rates of change that might rely more on memory. The results were consistent with this hypothesis. Besides supporting better performance, the fast condition was detected with a smaller change in brightness than was the slow rate, as reflected by a rate $\times$ amplitude interaction, $F(5,10)=5.7$, on normalized peck rates across these conditions. Holm-Sidak comparisons contrasting the two rates at each amplitude revealed that fast change supported better discrimination than did slow change at all amplitudes greater than $60 \mathrm{gsv} / \mathrm{s}$.

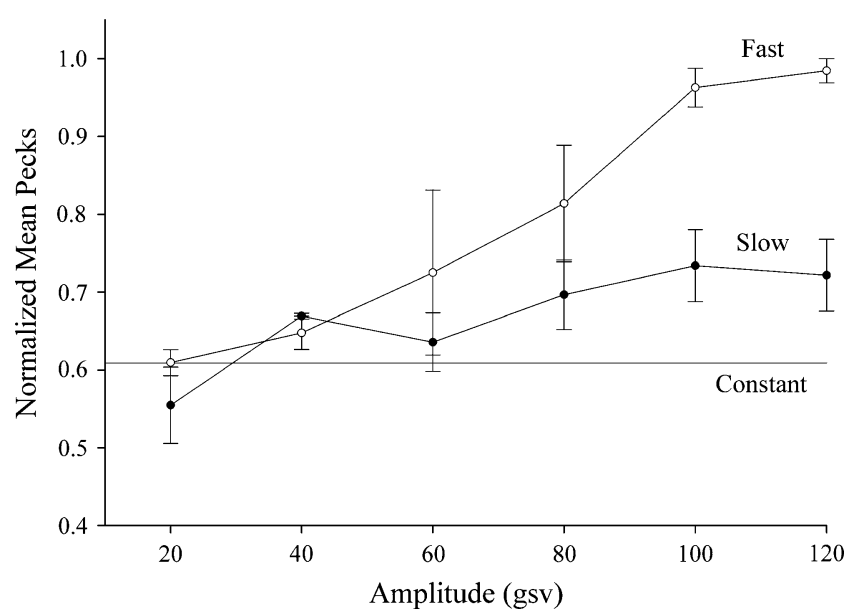

Fig. 5 Mean peck rates over each of six brightness ranges for fast ( $33.3 \mathrm{gsv} / \mathrm{s})$ and slow $(4.4 \mathrm{gsv} / \mathrm{s})$ rates of change. Error bars represent standard errors

\section{Discussion}

Our novel experimental approach showed that pigeons can discriminate between constant and dynamically changing brightness stimuli, indicating a capacity to detect and integrate experience over time. For rapidly changing stimuli, change detection influenced behavior within $8 \mathrm{~s}$ or less. Changes presented slower than $2 \mathrm{gsv} / \mathrm{s}$ were not readily detected within $60 \mathrm{~s}$. Overall, the time required for detecting change logarithmically increased with slower rates. Because adjacent changes in brightness were subliminal, mechanisms capable of comparing luminance at different time points are required for this discrimination. We suggest that pigeons may use a combination of perception and working memory mechanisms to make these comparisons.

For fast rates of change, they may have discriminated the changing and constant stimuli perceptually, perhaps using motion-related mechanisms. Because detecting motion and lighting changes are critical to flying birds (Crowder, Dawson, \& Wylie, 2003; Wang \& Frost, 1992), such mechanisms provide an easy explanation for the excellent change detection at the fastest rates. As detectable brightness changes began to stretch out over many seconds, temporal limits on such perceptual mechanisms would result in an inability to directly report "change," and the addition of working memory for the recent past would be needed. For these slower rates, the pigeons would ideally encode the earliest brightness value to compare subsequently with "now" to recognize that sufficient change had occurred.

Several lines of evidence suggest that multiple mechanisms might be involved. Experiment 2's results suggest that any perceptual mechanisms might be more sensitive to smaller changes in luminance than those used for judging slow changes. For the faster rates, the pigeons typically showed a linear response function, while constant trials over the same time range showed an exponentially decreasing response rate. As rates of change slowed, the response functions increasingly took on an exponential form, suggesting an increasing similarity to constancy. This transition may reflect the involvement of separate mechanisms (it may also reflect the increasing presence of a floor effect on response rates). Finally, Fig. 4 suggests a potential break point between these mechanisms. Over a large range of fast rates, the pigeons should be able to exhibit "change" behavior within a few seconds. Only with rates of 10 or fewer gsv/s did they begin to need substantially more time to detect a change. This change in the function may partially reflect the point in time at which memory starts to mediate the temporal comparisons.

Our results indicate that pigeons can continually update their memory to evaluate changes in the "recent past" for about $20-30$ s or so (see Fig. 4). This value converges with 
those established by memory tasks using discrete stimuli. Forgetting may be one reason that slow changes are so infrequently detected here. Constantly losing track of their best reference point for retroactively judging change would require encoding new comparison values mid-presentation.

One advantage of the present procedure is that no empty delay is involved in measuring memory. Concerns have been raised that the similarity of the memory delay with the intertrial interval complicates how pigeons respond in MTS procedures (Sherburne, Zentall, \& Kaiser, 1998). The dynamic nature of our procedure alleviates such problems, since the pigeons must be continually engaged with the task of detecting change. Regardless, our results show why so many predators stalk prey slowly: They are trying to approach outside of the temporal window over which prey can detect change by either detecting movement or comparing in memory two moments in time.

An issue to consider is that our fastest divergence times occurred after $4 \mathrm{~s}$. By this time, memory alone could have been involved, rather than perception. Our go/no-go task prevented us from seeing earlier differences in responding, since the birds required time to change the momentum of their initial pecking behavior. Future research with a choice task in which stimulus duration could be precisely controlled should help isolate the separate roles of perceptual and memory mechanisms. This task would also help us determine how amount and number of changes/ oscillations each contribute to change detection.

An additional reason the pigeons may have become poorer with slow changes could be related to limitations on vigilance or sustained attention. How birds concentrate on one task for extended periodsis not well investigated, but any reduction in attention over time would impair change detection. Furthermore, if our birds viewed the constant stimuli as aversive, they may have also oriented away from the screen (e.g., Wasserman, Franklin, \& Hearst, 1974). If this generalized to slow trials, this behavior would also decrease their chances of detecting change. Future experiments could reinforce pecks to the constant stimulus, thus prompting the birds to reduce pecking as soon as they detect change, instead of when they think change is not occurring. Despite these considerations, our novel approach to examining pigeons' experience of dynamic and continuous stimulation suggests that they are not creatures of the "now" but, more, creatures of the last minute or so. This limitation must dramatically influence how they structure and understand their experience of reality (e.g., Schmidt \& Cook, 2006).

It is important now to examine a variety of species and other dimensions (i.e., color, movement, shape), using continuous discriminations like this one. Integrative time horizons may vary across species, and this might be linked to encephalization, life span, natural history, and mobility.
Some animals, like humans, might be able to focus and retain information for extended periods of time, resulting in the detection of change at rates beyond those tolerated by our pigeons (e.g., allowing the linkage of seed planting to later harvesting). Investigating how different animals detect change should provide fundamental insights into the evolution of cognition, event detection, working memory, and intelligence and how these mechanisms function to produce coherent subjective realities.

Author Note This work was supported by Grant 0718804 from the National Science Foundation. E-mail: Robert.Cook@tufts.edu. Home Page: www.pigeon.psy.tufts.edu

\section{References}

Blough, D. S. (1959). Delayed matching in the pigeon. Journal of the Experimental Analysis of Behavior, 2, 151-160.

Cook, R. G., \& Blaisdell, A. P. (2006). Item memory in successive same-different discriminations. Behavioural Processes, 72, 255264.

Cook, R. G., Kelly, D. M., \& Katz, J. S. (2003). Successive two-item same-different discrimination and concept learning by pigeons. Behavioural Processes, 62, 125-144.

Crowder, N., Dawson, M., \& Wylie, D. (2003). Temporal frequency and velocity-like tuning in the pigeon accessory optic system. Journal of Neurophysiology, 90, 1829-1841.

Grant, D. S. (1976). Effect of sample presentation time on long-delay matching in the pigeon. Learning and Motivation, 7, 580-590.

Hodos, W., \& Bonbright, J. C., Jr. (1972). The detection of visual intensity differences by pigeons. Journal of the Experimental Analysis of Behavior, 18, 471-479.

James, W. (1890). The principles of psychology (Vol. 1). London: Holt.

Kopelman, M. D. (1987). Two types of confabulation. Journal of Neurology, Neurosurgery and Psychiatry, 50, 1482-1487.

Metzinger, T. (2003). Being no one: The self-model theory of subjectivity. Cambridge: MIT Press.

Rensink, R. A. (2002). Change detection. Annual Review of Psychology, $53,245-277$.

Roberts, W. (2006). Evidence that pigeons represent both time and number on a logarithmic scale. Behavioural Processes, 72, $207-$ 214.

Sacks, O. (1998). The man who mistook his wife for a hat and other clinical tales. New York: Touchstone.

Schmidt, G. F., \& Cook, R. G. (2006). Mind the gap: Means-end discrimination by pigeons. Animal Behaviour, 71, 599-608.

Seiffert, A. E., \& Cavanagh, P. (1999). Position-based motion perception for color and texture stimuli: Effects of contrast and speed. Vision Research, 39, 4172-4185.

Sherburne, L. M., Zentall, T. R., \& Kaiser, D. H. (1998). Timing in pigeons: The choose-short effect may result from pigeons' "confusion" between delay and intertrial intervals. Psychonomic Bulletin \& Review, 5, 516-522.

Tononi, G. (2004). An information integration theory of consciousness. BMC Neuroscience, 5, 42.

Wang, Y., \& Frost, B. J. (1992). Time to collision is signaled by neurons in the nucleus rotundus of pigeons. Nature, 356, 236238. 
Wasserman, E. A., Frank, A. J., \& Young, M. E. (2002). Stimulus control by same-versus-different relations among multiple visual stimuli. Journal of Experimental Psychology. Animal Behavior Processes, $28,347-357$.

Wasserman, E. A., Franklin, S. R., \& Hearst, E. (1974). Pavlovian appetitive contingencies and approach versus withdrawal to conditioned stimuli in pigeons. Journal of Comparative and Physiological Psychology, 86, 616-627.
Wixted, J. T. (1989). Nonhuman short-term memory: A quantitative reanalysis of selected findings. Journal of the Experimental Analysis of Behavior, 52, 409-426.

Wright, A. A., Santiago, H. C., Sands, S. F., Kendrick, D. F., \& Cook, R. G. (1985). Memory processing of serial lists by pigeons, monkeys, and people. Science, 229, 287-289.

Zihl, J., Von Cramon, D., \& Mai, N. (1983). Selective disturbance of movement vision after bilateral brain damage. Brain, 106, 313-340. 